\title{
Is Capillary Electrophoresis a New Tool to Monitor Acute Lithium Poisoning in Human?
}

Tsandni Jamal ${ }^{1}$, Carole Hennequin ${ }^{1}$, Rabah Gahoual ${ }^{2,3}$, Annie Leyris ${ }^{2}$, Jean-Louis Beaudeux ${ }^{1}$, Frédéric J. Baud ${ }^{4,5}$, and Pascal Houzé 1,2,3 $^{1,2}$

${ }^{1}$ Service de biochimie générale, Hôpital universitaire Necker-Enfants Malades, AP-HP, 149 rue de Sèvres, 75015 Paris, France

${ }^{2}$ Laboratoire de chimie analytique, Faculté de Pharmacie, Université Paris Descartes, 4 avenue de l'Observatoire, 75005 Paris, France

${ }^{3}$ Unité de Technologies Biologiques et Chimiques pour la Santé (UTCBS), Paris 5-CNRS UMR8258 Inserm U1022, Faculté de Pharmacie, Université Paris Descartes, Paris, France

${ }^{4}$ Assistance Publique - Hôpitaux de Paris, Département d'Anesthésie et de Réanimation, Adult Intensive Care Unit, Centre hospitalo-universitaire Necker - Enfants Malades, 149 rue de Sèvres, 75015 Paris, Université Paris Descartes, France

${ }^{5}$ EA7323 Evaluation of Therapeutics and Pharmacology in Perinatality and Pediatrics - Hôpitaux Universitaires Cochin - Broca - Hôtel Dieu, Site Tarnier, Université Paris Descartes, 75006 Paris, France

Corresponding author:

Pascal Houzé, PharmD, PhD,

Service de biochimie générale, Hôpital universitaire Necker-Enfants malades, AP-HP, 149 rue de Sèvres, 75015 Paris, France.

Email: pascal.houze@aphp.fr 
ABSTRACT: A 38-year-old man was admitted in the intensive care unit (ICU) after supposed ingestion of 504 sustainedrelease tablets of Theralithe ${ }^{\mathrm{TM}}$ corresponding $\sim 200 \mathrm{~g}$ of lithium carbonate. At the admission, $\sim 19.5 \mathrm{~h}$ after ingestion, the patient was conscious with trembling limbs, intense thirst, profuse sweats and vomiting and lithium serum concentration was $14.2 \mathrm{mmol} / \mathrm{L}$. Toxicological screenings performed in urine and serum, were negative. Patient was treated with continuous extrarenal epuration by continue veno-venous hemodiafiltration starting (CCVHDF) $24 \mathrm{~h}$ postadmission and was carried on until $64 \mathrm{~h}$. After 11 days in ICU, the patient was dismissed to the service without sequelae, and transferred to a psychiatric unit. To follow lithium concentrations in serum, urines and dialysates, we developed a simple, rapid and reliable method by capillary zone electrophoresis (CZE). Separation was achieved in $7 \mathrm{~min}$. The method was linear between 0.14 and 1.44 $\mathrm{mmol} / \mathrm{L}$ for serum samples, and between 0.07 and to $1.44 \mathrm{mmol} / \mathrm{L}$ for urines and dialysates. Limits of quantification were $0.15 \mathrm{mmol} / \mathrm{L}$ and $0.07 \mathrm{mmol} / \mathrm{L}$ for serum and others fluids, respectively. Intra- and inter-day precisions expressed as CV were systematically inferior to $12.1 \%$ for serum and $8.2 \%$ for other fluids. Results obtained regarding precision, accuracy, recovery and stability were satisfying, with recoveries ranging from 91.0 to $102.0 \%$. Serum, urine and dialysate samples were measured using CZE and flame photometry. We observed a strong correlation between both methods as assessed by linear regression and Bland-Altman analysis. For the intoxicated patient, the assay was successfully applied to serum, urine and dialysates to determine the amount of lithium present in circulation and excreted. Lithium amounts in dialysates were estimated to correspond to $89 \%$ of total lithium excreted during CCVHF session while urine excretion account only for $11 \%$. 


\section{INTRODUCTION}

Bipolar disorder is still a widespread psychopathic disorder in the world (1). Lithium salts have been used for decades in the treatment of this pathology (2). Their therapeutic efficacy has no longer to be proved and has been reported through numerous old and recent publications (1-3). Lithium is considered the most effective long-term therapy for bipolar disorder (1). It allows to prevent both depression and mania in addition to reduce the risk of suicide and shortterm mortality (4).

In return for its wide therapeutic use, lithium is still at the origin of numerous poisonings, mainly in a context of chronic drug accumulation, but self-administration of toxic doses may account for 20-27\% of the hospitalized poisoning (5, $6)$.

Serum lithium concentrations higher than $1.5 \mathrm{mmol} / \mathrm{L}$ are considered toxic and can induce severe renal, endocrine and neuronal toxicities (7-9). Specific treatment of lithium poisoning includes evacuator treatment like whole bowel irrigation, especially for sustained-release tablets $(10)$ and renal removal therapy for severe forms $(11,12)$. Using repeated hemodialysis (HD) sessions, concentration rebounds are usually observed at the end of HD session due to organs from extracellular fluids redistribution (13). Continuous extrarenal epuration by veno-venous hemodiafiltration (CCVHDF) is now frequently reported to treat severe lithium poisoning and better control of lithium concentration rebounds (5, $13)$.

Atomic emission spectrometry (AES) using flame photometer or ion-selective electrode, is currently used in laboratories to measure lithium concentrations $(14,15)$. Capillary electrophoresis is an analytical technique specially adapted for the separation of ions. This method has shown a great potential for separation and quantification of lithium and other ions in non-biological fluids (16) and biological fluids (17-19). This type of instrument can be commonly found in hospital toxicology or biochemistry department. Thus, CE has been implemented for the analysis of serum carbohydrate deficient transferrin to detect alcohol abuse and for the analysis of hemoglobin variants in order to identify $\alpha$ Thalassemias $(20,21)$. In a context of acute poisoning, we developed a simple, rapid and reliable method by capillary 
zone electrophoresis (CZE) to measure lithium concentrations in different biological (serums, urines) and non-biological fluids (dialysates). In order to fully assess the method, the results achieved by CZE were compared with those obtained by AES considered as the reference method.

\section{CASE HISTORY}

A 38-year-old man was admitted in the intensive care unit (ICU) at Necker-Enfants Malades hospital, after supposed ingestion of 504 sustained-release tablets of lithium carbonate (Theralithe LPTM), corresponding to 5.44 mol of lithium ion. At the admission, $16 \mathrm{~h}$ post-ingestion, the patient was conscious (Glascow score 15) with trembling limbs, intense thirst, profuse sweats and vomiting symptoms. Serum lithium concentration was measured at $14.2 \mathrm{mmol} / \mathrm{L}$. Toxicological screenings performed in urine and serum, were negative. Regarding sustained high-serum lithium concentrations, CCVHDF using Hemosol ${ }^{\mathrm{TM}}$ as dialysate solution, was started $16.5 \mathrm{~h}$ post-ingestion and continued to $63 \mathrm{~h}$ post-ingestion, corresponding to the duration of the CVVHDF session of $47 \mathrm{~h}$, until the return of lithium concentrations in the therapeutic zone. In addition to the CCVHDF session, the attending physician prescribed a 6-h session of intermittent HD $94 \mathrm{~h}$ postingestion owing to the plateau of serum lithium concentrations at $\sim 2.8 \mathrm{mmol} / \mathrm{L}$. The clinical evolution of the patient was characterized by an inhalation pneumopathy requiring intubation during 6 days, transient oliguria and sepsis shock with hypovolemia treated to cefazolin and noradrenaline. To follow lithium elimination, serums were sampled every 3, 6 and $12 \mathrm{~h}$. Urines were collected every $3 \mathrm{~h}$. During the CCVHDF session, a sample was taken from each change in the effluent bag. After 11 days in ICU, the patient was released from the unit without sequelae, and transferred to a psychiatric unit.

\section{MATERIALS AND METHODS}

Chemicals. Imidazole, tropolone, 18-crown-6-ether, ammonium acetate, potassium, sodium, calcium and magnesium as chloride salts and lithium carbonate, all with purities higher than 99\%, were purchased from Sigma-Aldrich (St QuentinFallavier, France). Acetonitrile, sodium hydroxidewere obtained from VWR International SAS (Fontenay-sous-Bois, France). Ultrapure water (18.2M $2 . \mathrm{cm}-1)$ was prepared by a Milli-Q System (Millipore, Molsheim, France). Lyphochek Quantitative Urine Control $^{\mathrm{TM}}$ (Bio-Rad, Marnes la Coquette, France) was used as free-lithium urine blank. Lithium-free blank serum was 
a gift of Etablissement Français du Sang, Necker-Enfants Malades (Paris, France). Hemosol ${ }^{\mathrm{TM}}$ solution was a gift of the Pharmacy department, Necker-Enfants Malades.

Preparation of reagents, calibrators and quality control samples. Electrophoretic buffer was obtained by dissolving imidazole $(5 \mathrm{mmol} / \mathrm{L})$, tropolone $(0.3 \mathrm{mmol} / \mathrm{L})$ and 18 -crown-6-ether $(0.45 \mathrm{mmol} / \mathrm{L})$ in ultrapure water. After complete dissolution, $\mathrm{pH}$ of the solution was adjusted to 4.15 using $1 \mathrm{mmol} / \mathrm{L}$ formic acid solution. Buffer was kept to $4^{\circ} \mathrm{C}$ and was stable during 2 months. Before use, buffer was degassed by sonication under reduced pressure and filtered through a $0.45-\mu \mathrm{m}-$ membrane (Polylabo, Strasbourg, France).

Aqueous ammonium acetate solution at the concentration to $1.7 \mathrm{mmol} / \mathrm{L}$ was used as Internal Standard (IS).

Stock solution containing $720 \mathrm{mmol} / \mathrm{L}$ of lithium was prepared in ultrapure water. Working solutions were prepared by dilutions in ultrapure water to achieve a concentration of $72 \mathrm{mmol} / \mathrm{L}$ for the working solution 1 (WS1) and a concentration of $36 \mathrm{mmol} / \mathrm{L}$ for the working solution 2 (WS2).

To prepare urine or Hemosol ${ }^{\mathrm{TM}}$ calibrators, appropriate dilution of lithium working solutions WS1 and WS2 were spiked in lithium free matrix (urine or Hemosol ${ }^{\mathrm{TM}}$ ) to obtain final concentrations corresponding to $0.07,0.14,0.36,0.72,1.08$ and $1.44 \mathrm{mmol} / \mathrm{L}$ in lithium.

For serum calibrators, lithium-free blank serum was spiked in the same manner to obtain calibrators corresponding to 0.14 , $0.36,0.72,1.08$ and $1.44 \mathrm{mmol} / \mathrm{L}$ of lithium.

For all matrices, quality controls (QCs) were prepared using a similar procedure and spiked independently from the calibration standards to achieve a concentration of $0.20 \mathrm{mmol} / \mathrm{L}$ for the QC1, $0.50 \mathrm{mmol} / \mathrm{L}$ for the QC2 and $1.20 \mathrm{mmol} / \mathrm{L}$ for the QC3.

Capillary zone electrophoresis analysis of lithium. The analyses were performed on a P/ACETM MDQ capillary electrophoresis system (Sciex, Villebon sur Yvette, France) equipped with a variable wavelength UV detector. A barefused-silica capillary (total length (L), $57 \mathrm{~cm}$; effective length (l), $50 \mathrm{~cm}$; internal diameter, $75 \mu \mathrm{m})$ was selected. The part of the capillary ensuring electrophoretic separation was maintained at a constant temperature $\left(20^{\circ} \mathrm{C}\right)$ by immersion in a coolant circulating in a cartridge with a rectangular detection window $(800 \times 100 \mu \mathrm{m})$. Electrophoretic separation 
was performed in normal polarity (input: anode, output: cathode) using the electrophoretic buffer previously described. Samples were injected in hydrodynamic mode under a pressure of $0.5 \mathrm{psi}$ applied to the inlet vial. Time of injection was ranging from 2 to $10 \mathrm{~s}$ depending on the sample concentrations. A constant voltage of $15 \mathrm{kV}$ was applied and indirect UV detection was performed using a wavelength of $214 \mathrm{~nm}$. Data were collected and analyzed by the 32 Karat $^{\mathrm{TM}}$ version 8.0 software (Sciex, Villebon sur Yvette, France). Between consecutive runs, the capillary was washed with $0.1 \mathrm{~mol} / \mathrm{L}$ sodium hydroxide $(0.5 \mathrm{~min})$, followed by ultrapure water $(0.5 \mathrm{~min})$ and a final $1 \mathrm{~min}$ equilibrium with electrophoretic buffer by applying 50 psi pressure at the injection end. At the end of the day, the capillary was rinsed with ultrapure water (2 min, $50 \mathrm{psi})$.

To measure lithium concentration in urine samples, $20 \mu \mathrm{L}$ of IS (ammonium acetate solution $1.7 \mathrm{mmol} / \mathrm{L}$ ) were added to $100 \mu \mathrm{L}$ of urinary standards, urinary QC and samples. After mixing, samples were directly injected in a capillary. If necessary, appropriate dilution of samples in free matrix were performed. Lithium concentrations in dialysates were measured in the same manner using standards and QC prepared in Hemosol ${ }^{\mathrm{TM}}$.

For serum, $100 \mu \mathrm{L}$ (calibrators, QCs, and samples) were introduced in Eppendorf tubes, and $20 \mu \mathrm{L}$ of IS was added to each. After mixing, $300 \mu \mathrm{L}$ of acetonitrile were added to each samples. All samples were vortexed 1 min, then centrifuged for $5 \mathrm{~min}$ at $15000 \mathrm{rpm}$. After centrifugation, supernatants were transferred to borosilicate glass tubes and evaporated at room temperature under nitrogen stream. Dry residues were dissolved in $50 \mu \mathrm{L}$ of ultrapure water and injected in capillary electrophoresis system under hydrodynamic mode.

For all matrices, lithium concentrations in unknown samples were calculated by interpolation of the ratio lithium area/ammonium area (IS) versus lithium calibrators on standard curves.

Atomic emission spectrometry. The analyses were performed on Unicam ${ }^{\mathrm{TM}} 919$ atomic emission spectrometer (AES; Thermoptik Corporation, Germany). Calibrators ranging from 0.07 to $1.44 \mathrm{mmol} / \mathrm{L}$ and CQs $(0.20,0.50$ and 1.20 $\mathrm{mmol} / \mathrm{L})$ were prepared in potassium chloride solution $(1 \mathrm{~g} / \mathrm{L})$. All unknown biological samples were diluted in potassium chloride solution at the appropriate dilutions. Absorbances of calibrators, CQ and unknown samples were measured at a wavelength of $670.8 \mathrm{~nm}$. Lithium concentrations in unknown samples were calculated by interpolation of the absorbance of these unknown samples versus standard curve. 
Validation procedure. The selectivity was tested on one blank borosilicate tube, 10 blank serum and 10 blank urine samples from denying any use of lithium salts.

To study linearity, five replicates for each calibration level for each matrix were analyzed. The linearity of each calibration curve was estimated by the least-square regression not weighted.

Lower limit of quantification (LLOQ) was defined as the lowest calibrator with an intra- and inter-day coefficient of variation lower than $20 \%$. Upper limit of quantification was determined by spiking $1.44 \mathrm{~mol} / \mathrm{L}$ standard by increasing volume of stock lithium solution $(720 \mathrm{mmol} / \mathrm{L})$.

Intra-day precision, expressed as relative standard deviation (RSD), was calculated analyzing the QC samples (0.20, $0.50,1.20 \mathrm{mmol} / \mathrm{L})$ in ten replicates; day-to-day precision was measured by analyzing the QC samples (10 replicates) on 10 different nonconsecutive days. Analytical accuracy was determined as the relative deviation of the average of calculated lithium concentrations of the QC samples from the corresponding nominal values and expressed to relative error (RE).

Recoveries in different matrices were analyzed on QC1 and QC2 samples spiked by increasing volume of stock lithium solution $(720 \mathrm{mmol} / \mathrm{L})$. QC spiked samples were analyzed in quintuplet using a freshly prepared calibration curves. Stability of lithium in different matrix was evaluated using three QC levels. QC samples were analyzed in quintuplet using a freshly prepared calibration curves, following three freeze-thaw cycles. For recovery and stability studies, results were expressed to RE from the nominal values.

Serum $(n=29)$, urines $(n=27)$ and dialysates $(n=14)$ were analyzed by CZE and flame photometry methods. The correlation between two techniques was assessed by the least-square regression, and Bland-Altman analysis using the plot of difference versus mean of the two method results (22). Average of the differences between the two methods \pm 1.96 SD defines limits of acceptability for the Bland-Altman test.

\section{RESULTS}

Under the above-described conditions, lithium migrated to the detector in $<5 \mathrm{~min}$ (total run time $7 \mathrm{~min}$ ). Lithium peak was baseline separated from the IS, i.e ammonium, but also from other cations with a flat line around them (Figure 1). 
All peaks are sharp with a calculated efficiency of about 120.000 plates per meter. Peaks present a symmetric or asymmetric shape depending of their migration rates. No interfering peaks could be observed in all electropherograms resulting of analyzing of blank borosilicate tube, 10 blank serums (Figure 1) and 10 blank urine samples. Relative migration time (ratio between lithium and IS migration times) precision was below $2 \%$ both in intra-day and day-today evaluation.

(a)

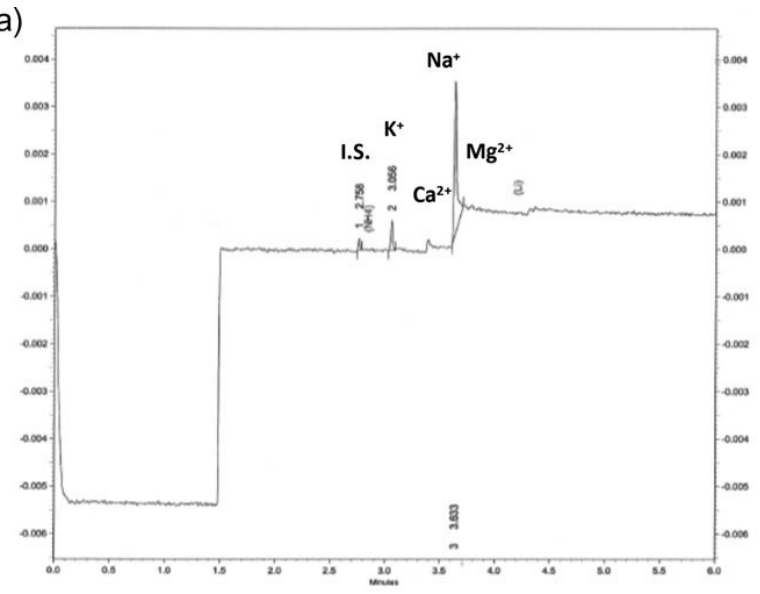

(c)

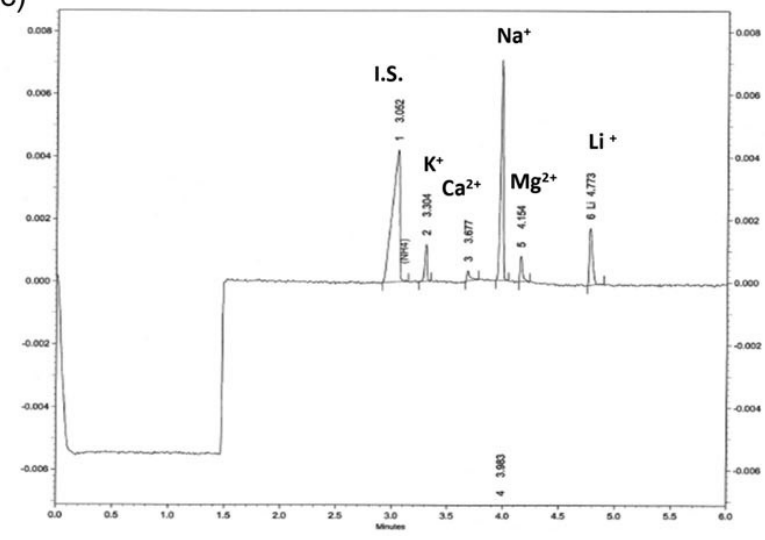

(b)

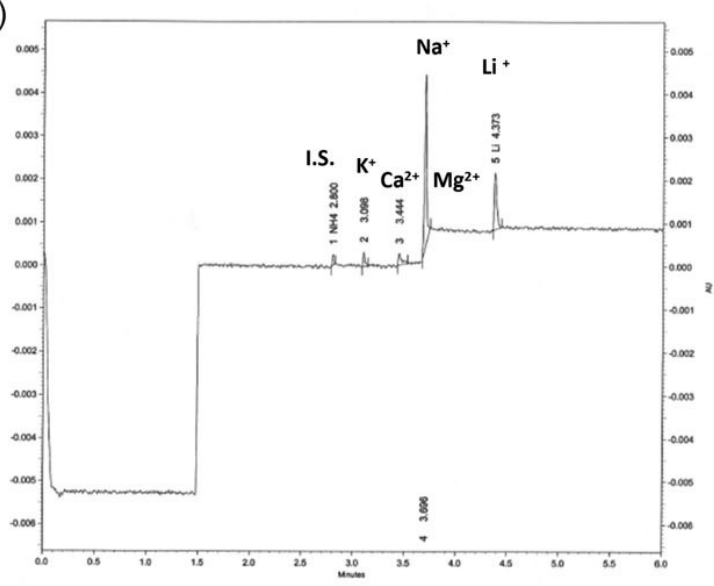

(d)

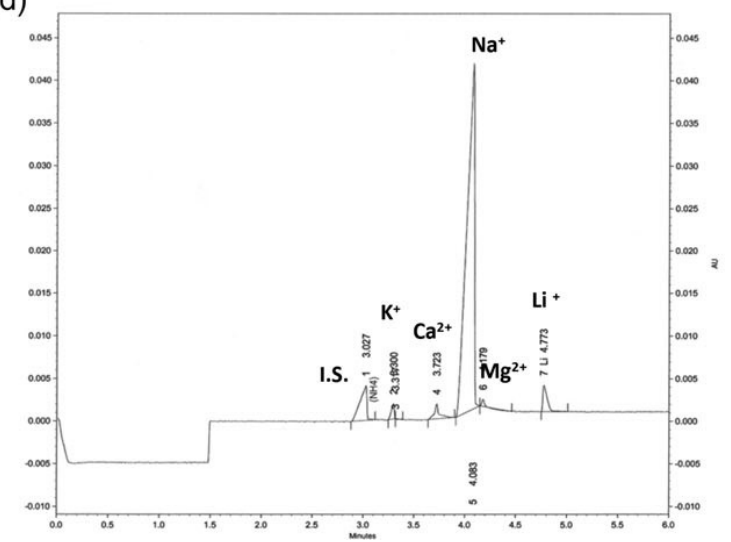

Figure 1. Electrophoregrams of a blank serum (a), serum (b), urine (c) and diafiltration effluents samples (d) obtained from poisoned patient $54 \mathrm{~h}$ after admission in intensive care unit. Electrophoregrams were obtained after appropriate dilutions for urine and diafiltration effluents according analytical process described in the text. Ammonium acetate was used as internal standard (IS). 
For urines and Hemosol ${ }^{\mathrm{TM}}$, calibration curves included six points and were linear in the range $0.07-1.44 \mathrm{mmol} / \mathrm{L}$. Serum calibration curve tested on five points $(0.14,0.36,0.72,1.08$ and $1.44 \mathrm{mmol} / \mathrm{L})$ was linear on this domain. The parameters of validation for all calibration curves are reported in Table I.

The limit of detection calculated as the lowest concentration producing a signal-to-noise ratio equal or higher than 3 , was $0.02 \mathrm{mmol} / \mathrm{L}$ for urines and Hemosol ${ }^{\mathrm{TM}}$, and $0.05 \mathrm{mmol} / \mathrm{L}$ for the serum. The LLOQs, calculated as the lithium concentration producing a RSD $\leq 20 \%$ was $0.07 \mathrm{mmol} / \mathrm{L}$ for urines and Hemosol ${ }^{\mathrm{TM}}$, and $0.15 \mathrm{mmol} / \mathrm{L}$ for serum. For all matrices, ULOQ was $4.32 \mathrm{mmol} / \mathrm{L}$.

For QC2 and QC3 samples in urines and Hemosol ${ }^{\mathrm{TM}}$, intra-day and day-to-day precision was systematically below 5\%. For QC1 which is closed to the LOQ, we obtained RSD values ranging from $6.4 \%$ to $8.2 \%$ for intra-day and dayto-day precision, respectively. In serum, RSD values were higher than those described in other matrices. They were below $10 \%$ except for QC1 showing intra-day and day-to-day precision equal to $10.5 \%$ and $12.1 \%$, respectively. For all matrices, accuracy values expressed as the RE, were ranging from 92.0 to $101.5 \%$. Regarding recoveries and stability studies, RE values ranged from $91.0 \%$ to $102.0 \%$.

\begin{tabular}{|c|c|c|c|}
\hline Matrice & Slope & Intercept & $\begin{array}{c}\text { Correlation coefficient } \\
\left(\mathbf{R}^{2}\right)\end{array}$ \\
\hline Serum & $0.856 \pm 0.010$ & $-0.062 \pm 0.008$ & $0.9803 \pm 0.055$ \\
\hline Urines & $1.305 \pm 0.008$ & $-0.016 \pm 0.005$ & $0.9979 \pm 0.022$ \\
\hline HemosolTM & $1.312 \pm 0.004$ & $-0.018 \pm 0.002$ & $0.9991 \pm 0.015$ \\
\hline
\end{tabular}

Table I. Linearity parameters for lithium in different matrices. Results are expressed as in mean value $\pm \operatorname{SD}(n=10)$

We compared our CZE method with AES using flame photometer. Figure 2 presents serum lithium values determined using both methods over time, and the values show good agreement between the methods. Figures $3 \mathrm{a}$ and $\mathrm{b}$ denoted a good correlation between the two analytical methods in serum, where 3a shows correlation described by the equation $\mathrm{y}(\mathrm{CZE})=1.1055 \mathrm{x}(\mathrm{AES})-0.1301 ; \mathrm{R} 2=0.9946$ and $3 \mathrm{~b}$ shows that the data are acceptable according to Bland - Altman analysis. Correlation and Bland-Altman analysis were performed for serum concentration values above CZE LOD, 
i.e., only for 24 samples. CZE was correlated to AES for 41 urine and dialysate samples, as reported by regression (Figure 3c) and Bland-Altman analysis (Figure 3d), although two values were outside of the Bland-Altman bands. We also used the CZE method to measure lithium amounts excreted in urines and during CCVHDF session (Figure 4).

\section{DISCUSSION}

Despite its toxicity and the development of new drugs less toxic such as lamotrigine (23), lithium is widely prescribed alone or associated to other drugs as valproate (24) to treat bipolar disorders. Lithium has a narrow therapeutic index and the recommended therapeutic serum lithium concentrations are in the range $0.6-1.0 \mathrm{mmol} / \mathrm{L}$ for classic tablets up to $1.2 \mathrm{mmol} / \mathrm{L}$ for sustained-release tablets (14). Therefore, lithium serum measurement is usually requested for Therapeutic Drug Monitoring (TDM) $(14,25)$, but can be also suitable for the management of poisoning. During the time course of acute poisoning, measurements of lithium concentrations in biological fluids as serum and urines, or nonbiological fluids (dialysate) can be required (i) to follow serum lithium kinetic; (ii) to measure eliminated amounts by different routes; (iii) to evaluate the efficiency of different therapeutic approaches such as CCVHDF (11). Lithium concentrations are commonly determined by flame photometry or specific electrode. The first remains the reference method to quantify lithium in biological samples directly or after mineralization. The use of a specific electrode is also usually reported to measure concentrations of lithium in serum and urines. Today, biochemistry laboratories no longer have a flame photometer, generally for safety reasons. On the other hand, specific electrodes are costly and sometimes lack specificity in particular for hemolysis or special samples (14). In biochemistry laboratories, serum lithium concentrations are routinely measured using colorimetric method on automatic clinical analyzers (26, 27). Although we have recently demonstrated that an automated colorimetric method could be used to dose lithium in different matrices, most commercialized colorimetric methods are not validated by manufacturer to measure lithium in other matrix than serum or plasma (28). However, ion detection and quantification can be achieved by separative methods such as ion chromatography or capillary electrophoresis (29-31). Capillary electrophoresis has a significant number of advantages compared to ion chromatography, such as shorter analysis time and smaller injected volumes, but often presented a 
limited sensitivity (32). These methods have been largely applied to quantify different ions in various application fields like water analysis $(16,33)$ or in medical and forensic applications $(19,34)$. Only one study reported the measurement of lithium concentration in biological fluids of animals (35). In human, monitoring of lithium concentration was previously reported using capillary isotachophoresis $(17,36)$, CZE (14) and microchip electrophoresis (18).

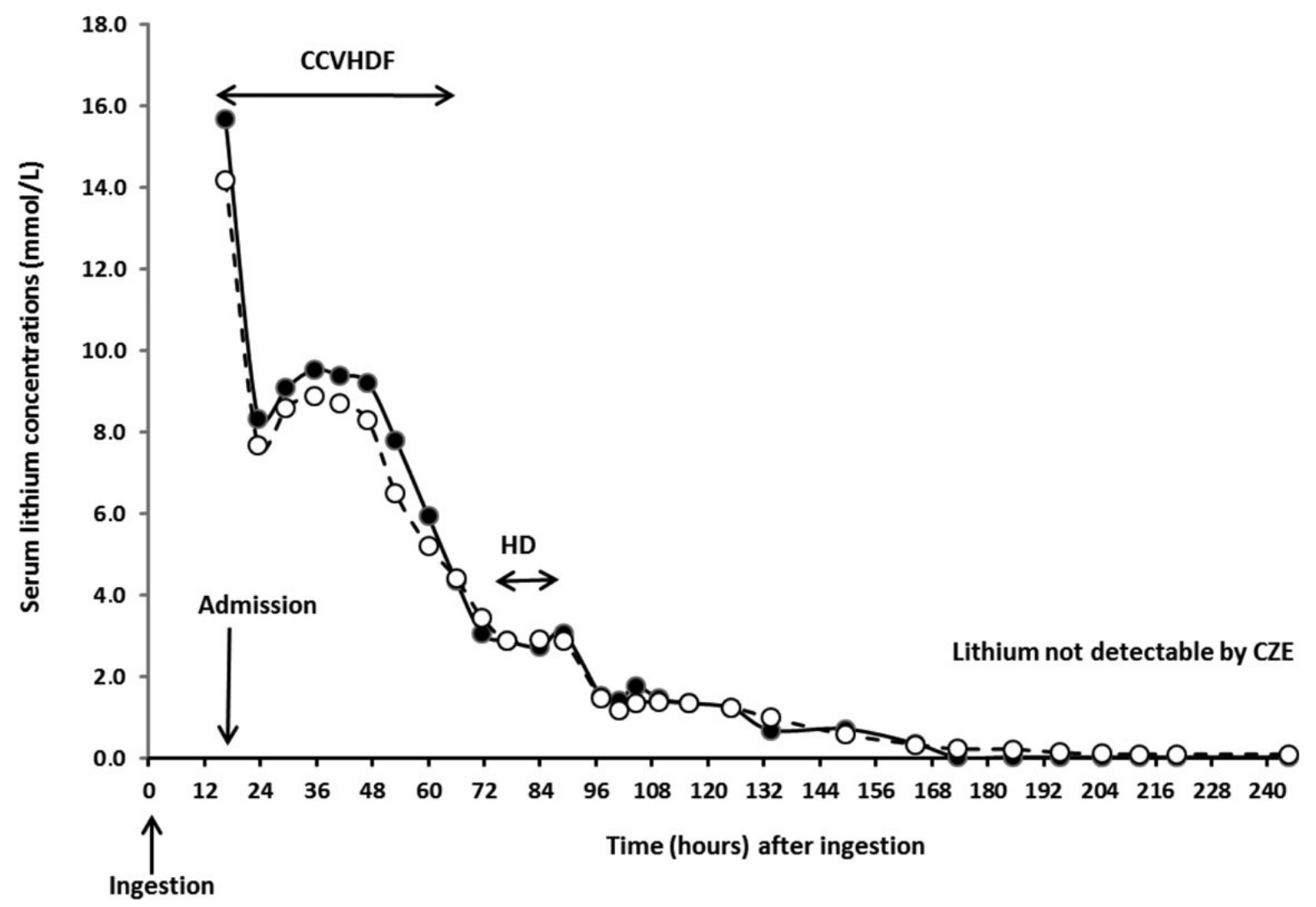

Figure 2. Time course of serum lithium concentrations determined by AES (white circles) and CZE (black circles). HD: hemodialysis; CVVHDF: continue venovenous hemodiafiltration; CZE: capillary zone electrophoresis.

In the context of acute intoxication, we have developed a CZE method to quantify lithium in different matrices. Our method allows a good separation of lithium from other cations $(\mathrm{K}+, \mathrm{Ca} 2+, \mathrm{Na}+$ and $\mathrm{Mg}++)$ commonly found in biological matrices and in Hémosol ${ }^{\mathrm{TM}}$. The ammonium ion is a physiological compound, and its use as an internal standard can therefore be questioned. However, its physiological serum concentrations is below $50 \mu \mathrm{mol} / \mathrm{L}$ and due to the sensitivity of our method, this concentration cannot be detected. Hémosol ${ }^{\mathrm{TM}}$ formulation does not contain ammonium. So 
for these two matrices, physiological ammonium concentration cannot induce variability in the response of the internal standard.

(a)

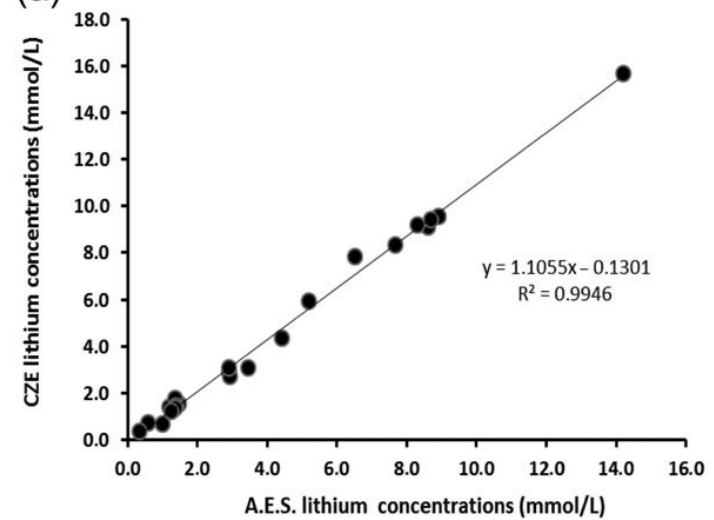

(c)

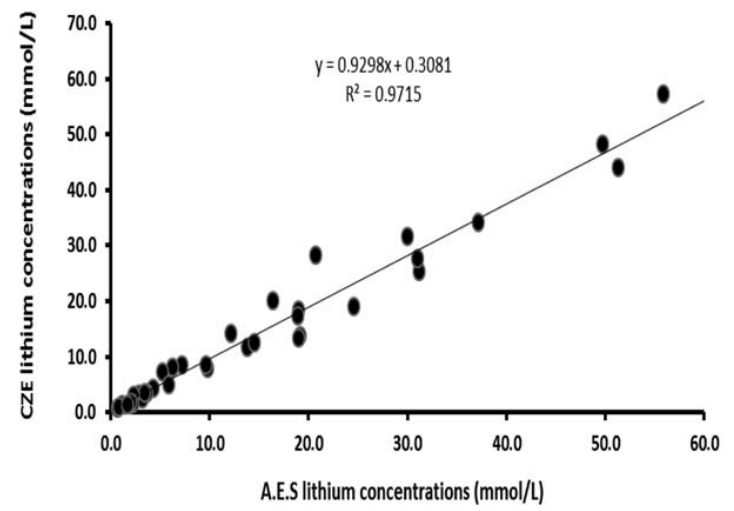

(b)

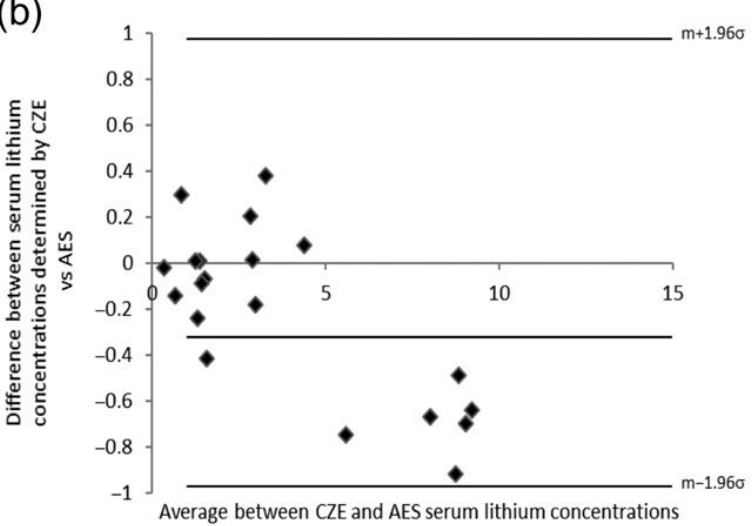

(d)

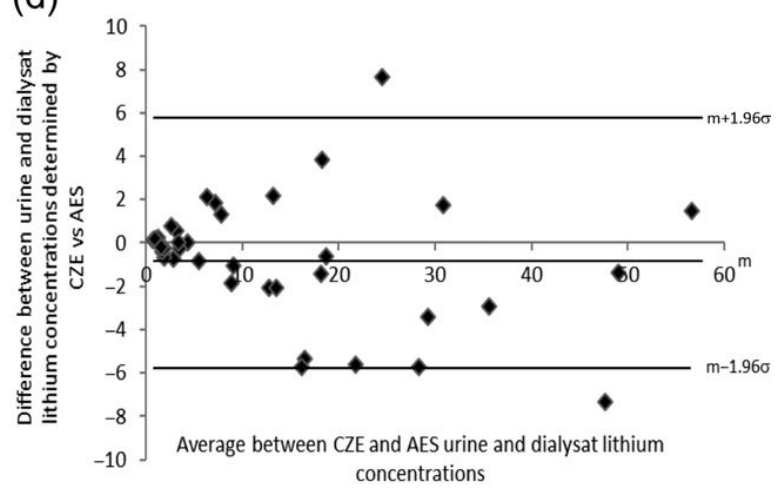

Figure 3. Correlation analysis for serum (a) and for urines and diafiltration effluents (c) samples between atomic emission spectrometry (AES) and capillary zone electrophoresis (CZE) methods. Bland-Altman analysis serum (b) and for urines and diafiltration effluents (d) samples between both methods.

In urines, physiological ammonium concentration is higher than in serum $(50 \mathrm{mmol} / \mathrm{L})$ and could be detectable as internal standard. To verify this was not occurring, we calculated the average internal peak area for the 23 urinary samples to be equal to $1,200 \pm 185$ and compared this with internal standard levels in serum and dialysate specimens, equal to $1,156 \pm 129$ ( $\mathrm{P}>0.05$, Student's t-test) and 1,243 \pm 187 ( $\mathrm{P}>0.05$, Student's t-test), respectively. Therefore, in the case of this patient, physiological ammonium urine concentration does not induce variability in the response of the internal standard. In these conditions, we can keep the same internal standard for lithium measurement in different 
matrices. In the future, it may be necessary to change the nature of internal standard to a non-physiological cation (e.g., barium, strontium), which have been previously reported in other methods (14).

Due to the excellent efficiency provided by the CZE separation, the total analysis time including injection and inbetween capillary flushing could be maintain under $10 \mathrm{~min}$ which is compatible with high-throughput analysis. The specificity of the method was assessed by the absence of lithium peak on the electropherograms of borosilicate tube, of non-treated subjects and any analyte interfering due to patient treatment. Our method is rapid, easy to put (diluteand shoot for urine and Hémosol ${ }^{\mathrm{TM}}$ ), and robust as proved by the stability of migration times.

The validation procedure was designed in order to be in agreement with the ICH guidelines regarding analytical methods assessment and validation (37). For the serum samples, the method demonstrated to be linear on a concentration domain including therapeutic and toxic concentrations. Linearity parameters were similar to the results reported to Pascali et al. (14) but our LOQ revealed to be two times lower than there. For the measurement of serum lithium, Pascali et al. (14) only performs a dilution of the serum in the internal standard solution. In our method, $100 \mu \mathrm{L}$ of serum sample are treated by acetonitrile to precipitate proteins. Acetonitrile phase containing lithium and other cations is evaporated to obtain a dry residue which is dissolved in $50 \mu \mathrm{L}$ of ultrapure water. In this way, lithium was concentrated by a factor of 2 . This sample preparation can explain that our method is twice as sensitive as that reported by Pascali et al. (14). Our LLOQ in serum is of the same order of magnitude than reported by Vrouwe et al. (18). Note, in the context of emergency medical care, the low volume of patient serum required to perform the analysis of lithium using the CZE method does not imply additional blood sampling. Therefore, it could be easy to incorporate the CZE analysis using a minimal fraction of the blood conventionally sampled to the patient to perform other routine tests. Also, due to the miniaturized properties of the CE instrumentation, the injection volume used is particularly low, up to $60 \mathrm{~nL}$. This characteristic offers, considering the volume of the sample, the ability to perform a reinjection of the same sample several dozens of time if necessary without running out of sample.

For urines and Hemosol ${ }^{\mathrm{TM}}$, linearity parameters and LOQs were similar for both matrices. These LOQs are two times lower than that of serum and comparable to those reported in water control study. This low value has allowed following 
lithium elimination during the patient's hospitalization. Results obtained for precision, accuracy, recovery and stability studies were satisfying.

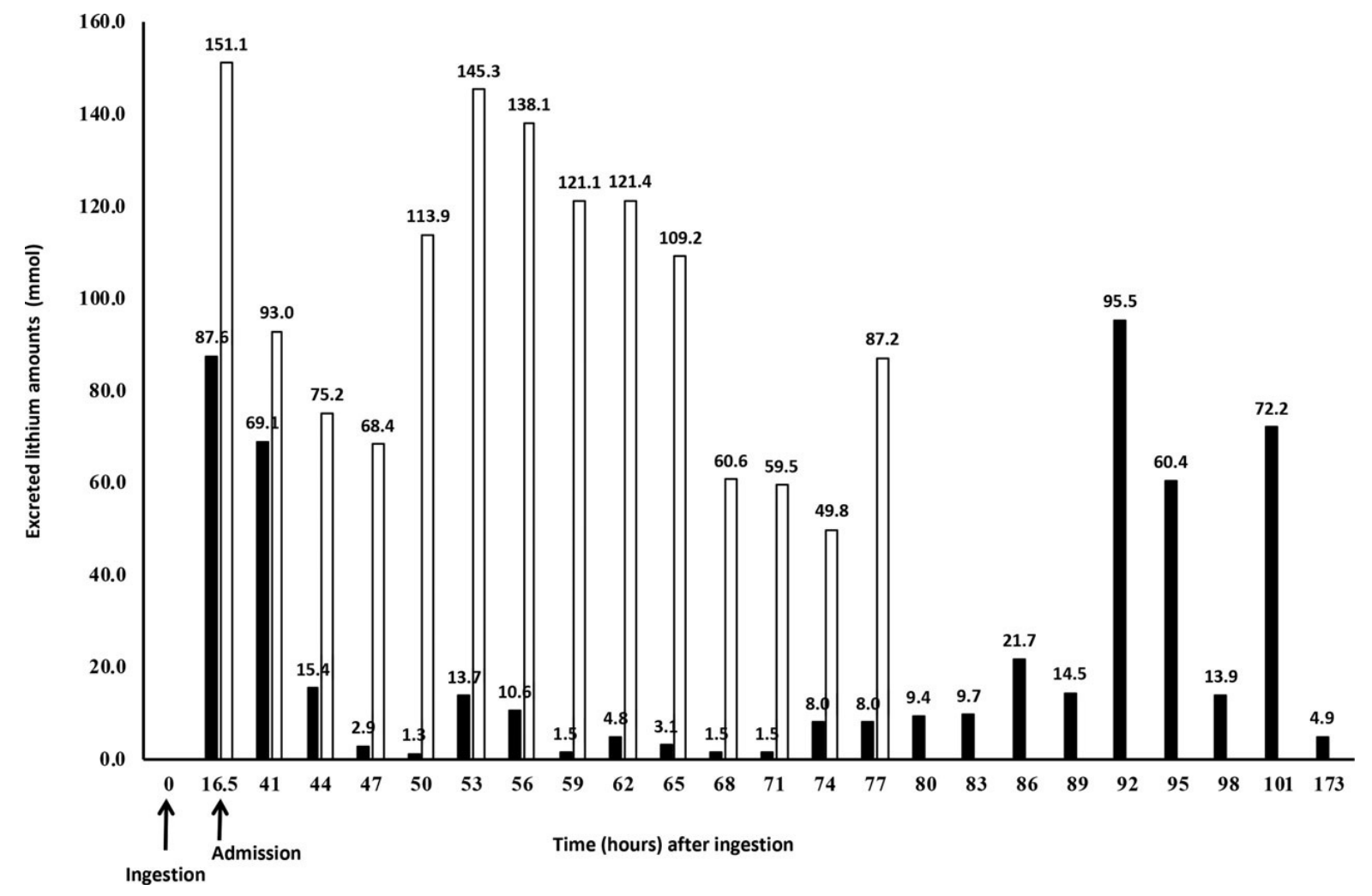

Figure 4. Amounts of lithium excreted in urines (black bars) and in diafiltration effluents (white bars). Number denoted lithium amounts determined by capillary zone electrophoresis (CZE).

We compared our CZE method with flame photometry. As reported in Figure 2, there was a strong correlation between the lithium plasma time courses determined by the two methods. Only five points at the end of plasma time course were below the LOQ of CZE. Regarding serum samples, both methods were well correlated (Figure 3a) and BlandAltman graphs showed that all samples were included in the limit of acceptability of the tests (Figure 3b). Good correlation was also reported in urines and diafiltration effluents (Figure 3c). Two urines samples were out limits of Bland-Altman test (Figure 3d), with observed bias that included both an underestimation or an overestimation values determined by the CZE method. These were observed for high urine concentration and did not appear clinically relevant. 
As reported in Figure 4, we followed urine and CCVHDF lithium excretions. Lithium amount in dialysates correspond to $90 \%$ of total lithium excreted during CCVHF session while urine excretion account only for $10 \%$ during the same period. Our results proved the great efficacy of CCVDF and confirmed that the best method to treat severe lithium acute poisoning without plasma rebounds $(5,12,13)$.

\section{CONCLUSION}

We report in this article, the development and statistical validation of a rapid, reliable, simple, reproducible, robust and economic CZE method to determine lithium concentration in different biological matrices. The results demonstrated the possibility to use the CZE method for the quantification of lithium in a concentration range relevant in the context of therapeutic use as well as acute intoxication for the different types of matrices considered. In addition, the CZE method performances demonstrated to be in agreement with the international standards regarding analytical method validation showing the method can be implemented to provide accurate lithium quantification in a matter of minutes. The method demonstrated its applicability to monitor lithium concentration for the reported case during an episode of acute poisoning. Indeed, the data obtained allowed during the intensive care process to determine the fraction of lithium successfully removed from the patient system over time, and finally identify the most effective therapeutic procedure. In laboratory equipped with capillary electrophoresis, our method showed to be a relevant and cost-effective alternative in addition to represent an orthogonal method to flame photometry as proved by closed correlation between reported in this paper. CZE can be especially adapted for analysis of unusual matrices as Hemosol ${ }^{\mathrm{TM}}$.

\section{INFORMED CONSENT}

No informed consent was necessary because CZE method was evaluated on samples necessary to toxicological following of patient and previously measured to AES.

\section{REFERENCES}

1. López-Muñoz, F., Shen, W.W., D’Ocon, P., Romero, A., Álamo, C. (2018) A history of the pharmacological treatment of bipolar disorder. International Journal of Molecular Sciences, 19. doi:10.3390/ijms19072143. 
2. Plotnikov, E.Y., Silachev, D.N., Zorova, L.D., Pevzner, I.B., Jankauskas, S.S., Zorov, S.D., et al. (2014) Lithium salts—simple but magic. Biochemistry (Mosc), 79, 740-749.

3. Gershon, S., Yuwiler, A. (1960) Lithium ion: a specific psychopharmacological approach to the treatment of mania. Journal of Neuropsychiatry, 1, 229-241.

4. Benard, V., Vaiva, G., Masson, M., Geoffroy, P.A. (2016) Lithium and suicide prevention in bipolar disorder. L'Encephale, 42, 234-241.

5. Zaworski, J., Delannoy, P.Y., Boussekey, N., Thellier, D., Georges, H., Leroy, O. (2017) Lithium: one drug, five complications. Journal of Intensive Care, 5, 70. doi:10.1186/s40560-017-0257-5.

6. Vodovar, D., El Balkhi, S., Curis, E., Deye, N., Mégarbane, B. (2016) Lithium poisoning in the intensive care unit: predictive factors of severity and indications for extracorporeal toxin removal to improve outcome. Clinical Toxicology (Phila), 54, 615-623.

7. Ott, M., Stegmayr, B., Salander Renberg, E., Werneke, U. (2016) Lithium intoxication: Incidence, clinical course and renal function - a populationbased retrospective cohort study. Journal of Psychopharmacology, 30, 1008-1019.

8. Gitlin, M. (2016) Lithium side effects and toxicity: prevalence and management strategies. International Journal of Bipolar Disorders, 4, 27-30.

9. Dineen, R., Bogdanet, D., Thompson, D., Thompson, C.J., Behan, L.A., McKay, A.P., et al. (2017) Endocrinopathies and renal outcomes in lithium therapy: impact of lithium toxicity. Quarterly Journal of Medicine, 110, 821-827.

10. Vodovar, D., Mégarbane, B. (2017) Do not forget gastrointestinal decontamination in the early management of lithium poisoning. Basic Clinical Pharmacology Toxicology, 120, 415-416.

11. Baird-Gunning, J., Lea-Henry, T., Hoegberg, L.C.G., Gosselin, S., Roberts, D.M. (2017) Lithium poisoning. Journal of Intensive Care Medicine, 32, 249-263.

12. Mirrakhimov, A.E., Barbaryan, A., Gray, A., Ayach, T. (2016) The role of renal replacement therapy in the management of pharmacologic poisonings. International Journal of Nephrology. doi:10.1155/2016/3047329.

13. Komaru, Y., Inokuchi, R., Ueda, Y., Nangaku, M., Doi, K. (2018) Use of the anion gap and intermittent hemodialysis following continuous hemodiafiltration in extremely high dose acute-on-chronic lithium poisoning: a case report. Hemodialysis International, 22, E15-E18.

14. Pascali, J.P., Sorio, D., Bortolotti, F., Tagliaro, F. (2010) Rapid determination of lithium in serum samples by capillary electrophoresis. Analytical and Bioanalytical Chemistry, 396, 2543-2546.

15. Lobeck, F. (1988) A review of lithium dosing methods. Pharmacotherapy, 8, 248-255.

16. Okamoto, H., Okamoto, Y., Hirokawa, T., Timerbaev, A.R. (2003) Trace ion analysis of sea water by capillary electrophoresis: determination of strontium and lithium pre-concentrated by transient isotachophoresis. The Analyst, 128, 1439-1442.

17. Okamoto, H., Timerbaev, A.R., Hirokawa, T. (2005) Simultaneous determination of metal ions, amino acids, and other small biogenic molecules in human serum by capillary zone electrophoresis with transient isotachophoretic

preconcentration. Journal of Separation Sciences, 28, 522-528. 18. Vrouwe, E.X., Luttge, R., Vermes, I., van den Berg, A. (2007) Microchip capillary electrophoresis for point-of-care analysis of lithium. Clinical Chemistry, 53, 117-123. 
19. Tagliaro, F., Bortolotti, F., Manetto, G., Cittadini, F., Pascali, V.L., Marigo, M. (2001) Potassium concentration differences in the vitreous humour from the two eyes revisited by microanalysis with capillary electrophoresis. Journal of Chromatography. A, 924, 493-498.

20. Schellenberg, F., Girre, C., Nalpas, B., Plat, A., Tome, A., Pagès, J.C. (2007) Analytical evaluation of a new capillary electrophoresis method for carbohydrate-deficient transferrin measurement. Clinica Chimica Acta, 382, 4853.

21. Jenkins, M., Ratnaike, S. (2003) Capillary electrophoresis of hemoglobin. Clinical Chemistry and Laboratory Medicine, 41, 747-754.

22. Bland, J.M., Altman, D.G. (1995) Comparing methods of measurement: why plotting difference against standard method is misleading. Lancet, 346, 1085-1087.

23. Bowden, C.L., Calabrese, J.R., Sach, G., Yatham, L.N., Behnke, K., Mehtonen, O.P., et al. In: Lamictal 606 Study Group. (2003) A placebocontrolled 18-months trial of lamotrigine and lithium maintenance treatment in recently manic or hypomanic patients with bipolar I disorder. Archives of General Psychiatry, 60, 392-400.

24. Geddes, J.R., Goodwin, G.M., Rendell, J., Azorin, J.M., Cipriani, A., Ostacher, M.J., et al. (2010) Lithium plus valproate combination therapy versus monotherapy for relapse prevention in bipolar I disorder (BALANCE): a randomised open-label trial. Lancet, 375, 385-395.

25. Guo, W., Guo, G.X., Sun, C., Zhang, J., Rong, Z., He, J., et al. (2013) Therapeutic drug monitoring of psychotropic drugs in China: a nationwide survey. Therapeutic Drug Monitoring, 35, 816-822.

26. Christenson, R.H., Mandichak, J.J., Duh, S.H., Augustyn, J.M., Thompson, J.C. (2003) Clinica1 performance characteristics of a new photometric lithium assay: a multicenter study. Clinica Chimica Acta, 327, 157-164.

27. Gruson, D., Lallali, A., Furlan, V., Taburet, A.M., Legrand, A., Conti, M. (2004) Evaluation of a new lithium colorimetric assay performed on the Dade Behring Dimension X-pand system. Clinical Chemistry and Laboratory Medicine, 42, 1066-1068.

28. Hennequin, C., Jamal, T., Leyris, A., Beaudeux, J.L., Houzé, P. (2018) Assessment of Architect cSystems Abbott® for the colorimetric measurement of lithium in urines and dialysates. Clinical Chemistry and Laboratory Medicine. doi:10.1515/cclm-2018-0175.

29. Pascali, J.P., Bortolotti, F., Tagliaro, F. (2012) Recent advances in the application of CE to forensic sciences, an update over years 2009-2011. Electrophoresis, 33, 117-126.

30. Zeng, W., Chen, Y., Cui, H., Wu, F., Zhu, Y., Fritz, J.S. (2006) Singlecolumn method of ion chromatography for the determination of common cations and some transition metals. Journal of Chromatography. A, 1118, 68-72.

31. Malavolta, M., Piacenza, F., Basso, A., Giacconi, R., Costarelli, L., Pierpaoli, S., et al. (2012) Speciation of trace elements in human serum by micro anion exchange chromatography coupled with inductively coupled plasma mass spectrometry. Analytical Biochemistry, 421, 16-25.

32. Coufal, P., Pacáková, V., Stulík, K. (2007) An evaluation of the experimental approaches to detection of small ions in CE. Electrophoresis, 28, 3379-3389.

33. Foret, F. (2009) Capillary electrophoresis of small ions using complex formation and indirect detection. Electrophoresis, 30, S34-S39. 
34. Lemos, N.P., Bortolotti, F., Manetto, G., Anderson, R.A., Cittadini, F., Tagliaro, F. (2001) Capillary electrophoresis: a new tool in forensic medicine and science. Science \& Justice, 41, 203-210.

35. Creson, T.K., Monaco, P.J., Rasch, E.M., Hagardorn, A.H., Ferslew, K. E. (1998) Capillary ion analysis of lithium concentrations in biological fluids and tissues of Poecilia (teleost). Electrophoresis, 19, 3018-3021.

36. Valásková, I1, Balázová, J, Havránek, E (1995) Monitoring of lithium levels in human serum after therapy with lithium preparations by capillary isotachophoresis. Journal of Chromatography. B, Biomedical Sciences and Applications, 674, 310-313.

37. Guideline, I.H.T. (2005). Validation of analytical procedures: text and methodology Q2 (R1). In International Conference on Harmonization, Geneva, Switzerland, 11-12. 